\title{
New Constraints on Neutrino Masses from Cosmology
}

\author{
Alessandro Melchiorri ${ }^{1}$, Scott Dodelson ${ }^{2}$, Paolo Serra ${ }^{1}$ and Anže Slosar ${ }^{3}$ \\ 1 Physics Department and sezione INFN, University of Rome "La Sapienza", Ple \\ Aldo Moro 2, 00185 Rome, Italy \\ 2 Particle Astrohysics Center, FERMILAB, Batavia, IL 60510-0500 \\ 3 Faculty of Mathematics and Physics, University of Ljubljana, 1000 Ljubljana, \\ Slovenia \\ E-mail: alessandro.melchiorri@roma1.infn.it
}

\begin{abstract}
By combining data from cosmic microwave background (CMB) experiments (including the recent WMAP third year results), large scale structure (LSS) and Lyman$\alpha$ forest observations, we derive upper limits on the sum of neutrino masses of $\Sigma m_{\nu}<0.17 \mathrm{eV}$ at $95 \%$ c.l.. We then constrain the hypothesis of a fourth, sterile, massive neutrino. For the 3 massless +1 massive neutrino case we bound the mass of the sterile neutrino to $m_{s}<0.26 \mathrm{eV}$ at $95 \%$ c.l.. These results exclude at high significance the sterile neutrino hypothesis as an explanation of the LSND anomaly. We then generalize the analysis to account for active neutrino masses (which tightens the limit to $m_{s}<0.23 \mathrm{eV}$ and the possibility that the sterile abundance is not thermal. In the latter case, the contraints in the (mass, density) plane are nontrivial. For a mass of $>1 \mathrm{eV}$ or $<0.05 \mathrm{eV}$ the cosmological energy density in sterile neutrinos is always constrained to be $\omega_{\nu}<0.003$ at $95 \%$ c.l.. However, for a sterile neutrino mass of $\sim 0.25 \mathrm{eV}, \omega_{\nu}$ can be as large as 0.01 .
\end{abstract}

Key words: cosmology, theory, cosmic microwave background, neutrinos

\section{Introduction}

Cosmological observations have started to provide valuable upper limits on absolute neutrino masses (see, e.g., the reviews $(1 ; 2)$ ), competitive with those from laboratory experiments. In particular, the combined analysis of highprecision data from Cosmic Microwave Background (CMB) anisotropies and Large Scale Structures (LSS) has already reached a sensitivity of $O(\mathrm{eV})$ (see, 
e.g., $(3 ; 4))$ for the sum of the neutrino masses $\Sigma$,

$$
\Sigma=m_{1}+m_{2}+m_{3}
$$

We recall that the total neutrino energy density in our Universe, $\Omega_{\nu} h^{2}$ (where $h$ is the Hubble constant normalized to $H_{0}=100 \mathrm{~km} \mathrm{~s}^{-1} \mathrm{Mpc}^{-1}$ ) is related to $\Sigma$ by the well-known relation $\Omega_{\nu} h^{2}=\Sigma /(93.2 \mathrm{eV})(5)$, and plays an essential role in theories of structure formation. It can thus leave key signatures in LSS data (see, eg.,(6)) and, to a lesser extent, in CMB data (see, e.g.,(7)). Very recently, it has also been shown that accurate Lyman- $\alpha(\operatorname{Ly} \alpha)$ forest data (8), taken at face value, can improve the current CMB +LSS constraints on $\Sigma$ by a factor of $\sim 3$, with important consequences on absolute neutrino mass scenarios $(37)$.

On the other hand, atmospheric, solar, reactor and accelerator neutrino experiments have convincingly established that neutrinos are massive and mixed. World neutrino data are consistent with a three-flavor mixing framework (see (10) and references therein), parameterized in terms of three neutrino masses $\left(m_{1}, m_{2}, m_{3}\right)$ and of three mixing angles $\left(\theta_{12}, \theta_{23}, \theta_{13}\right)$, plus a possible CP violating phase $\delta$.

Neutrino oscillation experiments are sensitive to two independent squared mass difference, $\delta m^{2}$ and $\Delta m^{2}$ (with $\delta m^{2} \ll \Delta m^{2}$ ), hereafter defined as (11)

$$
\left(m_{1}^{2}, m_{2}^{2}, m_{3}^{2}\right)=\mu^{2}+\left(-\frac{\delta m^{2}}{2},+\frac{\delta m^{2}}{2}, \pm \Delta m^{2}\right)
$$

where $\mu$ fixes the absolute neutrino mass scale, while the cases $+\Delta m^{2}$ and $-\Delta m^{2}$ identify the so-called normal and inverted neutrino mass hierarchies, respectively. Neutrino oscillation data indicate that $\delta m^{2} \simeq 8 \times 10^{-5} \mathrm{eV}^{2}$ and $\Delta m^{2} \simeq 2.4 \times 10^{-3} \mathrm{eV}^{2}$. They also indicate that $\sin ^{2} \theta_{12} \simeq 0.3, \sin ^{2} \theta_{23} \simeq 0.5$, and $\sin ^{2} \theta_{13} \leq$ few $\%$. However, they are currently unable to determine the mass hierarchy $\left( \pm \Delta m^{2}\right)$ and the phase $\delta$, and are insensitive to the absolute mass parameter $\mu$ in Eq. (2).

The absolute neutrino mass scale can also be probed by non-oscillatory neutrino experiments. The most sensitive laboratory experiments to date have been focussed on tritium beta decay and on neutrinoless double beta decay. Beta decay experiments probe the so-called effective electron neutrino mass $m_{\beta}(12)$,

$$
m_{\beta}=\left[c_{13}^{2} c_{12}^{2} m_{1}^{2}+c_{13}^{2} s_{12}^{2} m_{2}^{2}+s_{13}^{2} m_{3}^{2}\right]^{\frac{1}{2}}
$$


where $c_{i j}^{2}=\cos ^{2} \theta_{i j}$ and $s_{i j}^{2}=\sin ^{2} \theta_{i j}$. Current experiments (Mainz (13) and Troitsk (14)) provide upper limits in the range $m_{\beta} \leq$ few eV (5).

Neutrinoless double beta decay $(0 \nu 2 \beta)$ experiments are instead sensitive to the so-called effective Majorana mass $m_{\beta \beta}$ (if neutrinos are Majorana fermions),

$$
m_{\beta \beta}=\left|c_{13}^{2} c_{12}^{2} m_{1}+c_{13}^{2} s_{12}^{2} m_{2} e^{i \phi_{2}}+s_{13}^{2} m_{3} e^{i \phi_{3}}\right|
$$

where $\phi_{2}$ and $\phi_{3}$ parameterize relative (and unknown) Majorana neutrino phases (15). All $0 \nu 2 \beta$ experiments place only upper bounds on $m_{\beta \beta}$ (the most sensitive being in the $\mathrm{eV}$ range, with the exception of the Heidelberg-Moscow experiment (16), which claims a positive (but highly debated) $0 \nu 2 \beta$ signal $m_{\beta \beta}>0.17 \mathrm{eV}$ at $95 \%$ c.l. and corresponding to $m_{\beta \beta}$ in the sub-eV range at best fit $(17 ; 18)$.

Results from the Liquid Scintillator Neutrino Detector (LSND) (19) challenge the simplicity of the 3-flavour neutrinos picture. The LSND experiment reported a signal for $\bar{\nu}_{\mu} \rightarrow \bar{\nu}_{e}$ oscillations in the appearance of $\bar{\nu}_{e}$ in an originally $\bar{\nu}_{\mu}$ beam. To reconcile the LSND anomaly with results on neutrino mixing and masses from atmospheric and solar neutrino oscillation experiments, one needs additional mass eigenstates. The simplest possibility is that these additional states are related to right-handed neutrinos, for which bare mass terms $\left(M \nu_{R} \nu_{R}\right)$ are allowed by all symmetries. These would are sterile, i.e. not present in $S U(2)_{L} \times U(1)_{\gamma}$ interactions. The " $3+1$ sterile" neutrino explanation assumes that the $\bar{\nu}_{\mu} \rightarrow \bar{\nu}_{e}$ oscillation goes through $\bar{\nu}_{\mu} \rightarrow \bar{\nu}_{s} \rightarrow \bar{\nu}_{e}$. The additional sterile state is separated by the three active states by a mass scale in the range of $0.6 \mathrm{eV}^{2}<\Delta \mathrm{m}_{\mathrm{LSND}}^{2}<2 \mathrm{eV}^{2}$.

In these proceedings we will briefly review the current cosmological constraints on neutrino masses in the 3 and $3+1$ scenarios and compare them with the findings of $m_{\beta \beta}$ Heidelberg-Moscow and LSND experiments. The results presented here are mostly taken from (20) and (21) and we refer the reader to those papers for more details about the analyses.

\section{Cosmological Constraints on Neutrino Masses}

\section{Active Neutrino Scenario}

The method we adopt is based on the publicly available Markov Chain Monte Carlo package cosmomc (22). We sample the following eight-dimensional set of cosmological parameters, adopting flat priors on them: the physical baryon, CDM and massive neutrinos densities, $\omega_{b}=\Omega_{b} h^{2}, \omega_{c}=\Omega_{c} h^{2}$ and $\Omega_{\nu} h^{2}$, the ratio of the sound horizon to the angular diameter distance at decoupling, 
$\theta_{s}$, the scalar spectral index, the overall normalization of the spectrum $\mathrm{d} A$ at $k=0.05 \mathrm{Mpc}^{-1}$ and, finally, the optical depth to reionization, $\tau$. Furthermore, we consider purely adiabatic initial conditions and we impose flatness.

We include the three-year data (23) (temperature and polarization) with the routine for computing the likelihood supplied by the WMAP team and available at the LAMBDA web site. ${ }^{1}$ We marginalize over the amplitude of the Sunyaev-Zel'dovich signal, but the effect is small: including/excluding the correction change our conclusions on the best fit value of a single parameter by less than $2 \%$ and always well inside the $1 \sigma$ confidence level. We treat beam errors with the highest possible accuracy (see (24), appendix A.2), using full off-diagonal temperature covariance matrix, Gaussian plus lognormal likelihood, and fixed fiducial $C_{\ell}$ 's. The MCMC convergence diagnostics is done throught the Gelman and Rubin "variance of chain mean" / "mean of chain variances" $R$ statistic for each parameter. Our $2-D$ constraints are obtained after marginalization over the remaining "nuisance" parameters, again using the programs included in the cosmomc package. In addition to the CMB data, we also consider different datasets. We therefore consider the following cases:

- 1) WMAP-only: Only temperature, cross polarization and polarization WMAP data are considered. Plus a top-hat age prior 10 Gyrs $<t 0<$ 20Gyrs.

- 2) WMAP+SDSS: We combine the WMAP data with the the real-space power spectrum of galaxies from the Sloan Digital Sky Survey (SDSS) (31). We restrict the analysis to a range of scales over which the fluctuations are assumed to be in the linear regime $\left(k<0.2 h^{-1} \mathrm{Mpc}\right)$ and we marginalize over a bias $b$ considered as an additional nuisance parameter.

- 3) WMAP+SDSS+SN $\mathbf{S N}_{\text {Riess }}+\mathbf{H S T}+\mathbf{B B N}$ : We combine the data considered in the previous case with HST measurement of the Hubble parameter $h=0.72 \pm 0.07$ (25), a Big Bang Nucleosynthesis Prior of $\Omega_{b} h^{2}=$ $0.020 \pm 0.002$ and we finally incorporate constraints obtained from the SNIa luminosity measurements of (26) using the so-called GOLD data set.

- 4) $\mathbf{C M B}+\mathbf{L S S}+\mathbf{S N}_{\text {Astier }}$ : Here we include WMAP and also consider the small-scale CMB measurements of CBI (28), VSA (29), ACBAR (30) and BOOMERANG-2k2 (27). In addition to the CMB data, we include the constraints on the real-space power spectrum of galaxies from the SLOAN galaxy redshift survey (SDSS) (31) and $2 \mathrm{dF}$ (32). and the Supernovae Legacy Survey data from (33).

- 5) CMB+LSS+SN+BAO: We include in the previous case the constraints from the Baryonic Acoustic Oscillations (BAO) detected in the Luminous Red Galaxies sample of the SDSS (34).

- 6) CMB+SDSS+SN+Lyman- $\alpha$ : We include measurements of the small scale primordial spectrum from Lyman-alpha forest clouds $(35 ; 36)$ but we

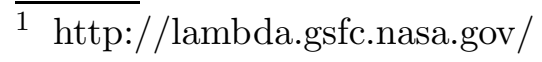




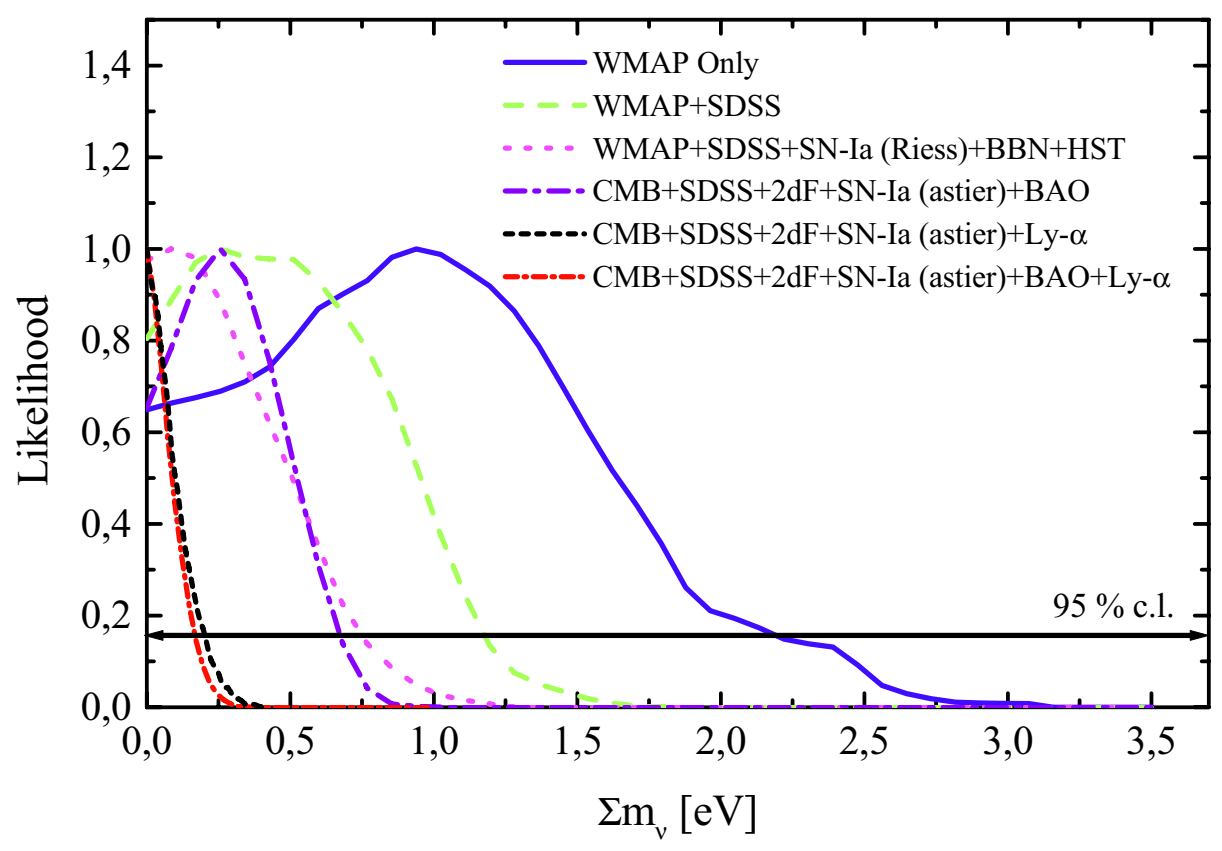

Fig. 1. Likelihood distributions for $\Sigma$ for the several analyses considered (see text).

don't consider BAO. The details of the analysis are the same as those in (37).

- 7) $\mathbf{C M B}+$ SDSS+BAO+Lyman- $\alpha$ : We also include BAO measurements to the previous dataset. Again, see (37) for more details.

In Fig. 1 we plot the likelihood distributions for $\Sigma$ for each of our analysis. As we can see, these curves do not show evidence for a neutrino mass and provide the $2 \sigma$ constraints reported in Table I. Such bounds are in good agreement with previous results in similar analyses $((23),(37))$ and we can clearly derive the following conclusions:

- As already showed in (23) and (38), the WMAP data alone, in the framework of cosmological models we are considering, is able to constrain $\Sigma<2.32 \mathrm{eV}$ at $95 \%$ c.l.. This limit should be considered as the most conservative since it relies on a single dataset.

- Inclusions of galaxy clustering and SN-Ia data has the ability of further constraining the results. The datasets used in the compilation 2, 3, 4 and 5 provide constraints of $\Sigma<1.12 \mathrm{eV}, \Sigma<0.77 \mathrm{eV}, \Sigma<0.72 \mathrm{eV}$ and $\Sigma<$ $0.68 \mathrm{eV}$ at $95 \%$ c.l. respectively. Those results are in agreement with the findings of (23). Different galaxy clustering and supernovae data have been used in order to identify the impact of possible systematics.

- Including SDSS Lyman- $\alpha$ data in 6 and 7 as in (37)) greatly improves the constraints on $\Sigma$ up to $\Sigma<0.21 \mathrm{eV}$ and $\Sigma<0.17 \mathrm{eV}$ (95\% c.l.). This result has important consequences for our analyses especially when compared with the $m_{\beta \beta}$ claim. However, we remark that the constraints on the linear density 


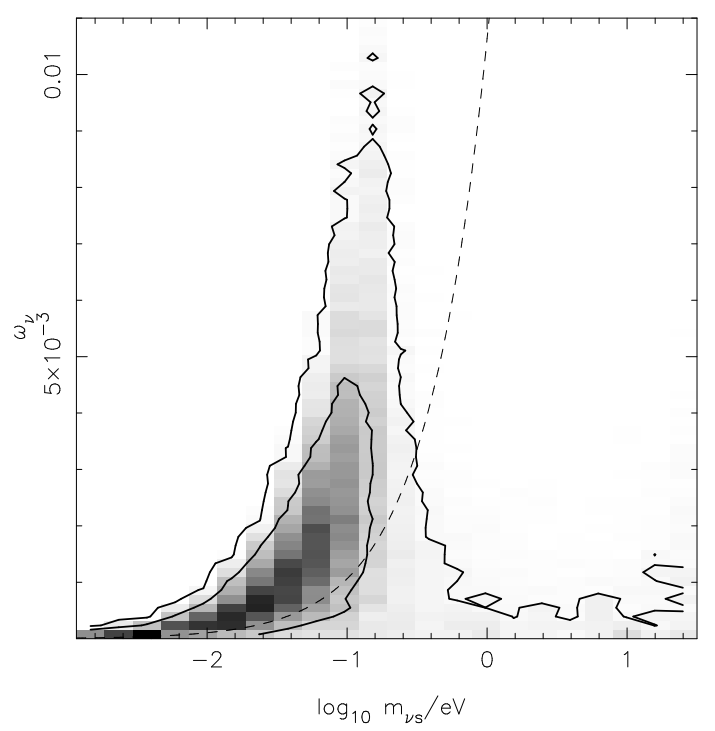

Fig. 2. 1,2- $\sigma$ constraints on the sterile neutrino mass and abundance.

fluctuations obtained from this dataset are derived from measurement of the Ly $\alpha$ flux power spectrum $P_{F}(k)$ after a long inversion process, which involves numerical simulations and marginalization over the several parameters of the Ly $\alpha$ model. This limit should therefore considered at the same time as the less conservative of the bunch.

\section{$3+1$ Sterile Neutrino Scenario}

Let us now derive constraints on neutrino masses in the case of a $3+1$ sterile scenario using all the cosmological datases presented above. If the active neutrino masses are fixed to zero and the sterile neutrino abundance is thermal the upper limit on the sterile neutrino mass is $0.26 \mathrm{eV}$ (all at $95 \%$ c.l.). Of course the active neutrino masses are not zero. Taking them as a free parameter leads to an upper limit on the sterile neutrino mass of $0.23 \mathrm{eV}$. This is tighter than the $m_{\nu}=0$ constraint because the limit is really on the sum of all neutrino masses. Fixing the active masses to zero allows the maximum $m_{s}$. Relaxing this restriction leaves less room for a large $m_{s}$. We have found some sensitivity to the mass difference of the sterile and active states (and this might be measurable with future data), but current data really constrain only the sum of all neutrino masses.

We now generalize further and allow the sterile neutrino abundance $\omega_{s}=\Omega_{s} h^{2}$ to vary. Fig. 2 shows the constraints in the $\omega_{s}-m_{s}$ plane. Note the distinct peak around the region of $m_{s} \sim 0.25 \mathrm{eV}$, presenting an allowed region of parameter space with anomalously large values of $\omega_{s}$. To the left of this peak, the equivalence epoch, $a_{\mathrm{EQ}}$, is very large and the resulting Integrated Sachs Wolfe effect precludes agreement with CMB data. When $m_{s}$ is in the allowed regime, $a_{\mathrm{EQ}}$ would still be too large were $\omega_{m}$ fixed. However, a model with larger $\omega_{m}(\sim 0.18)$ leads to an even smaller, acceptable $a_{\mathrm{EQ}}$. Fortu- 

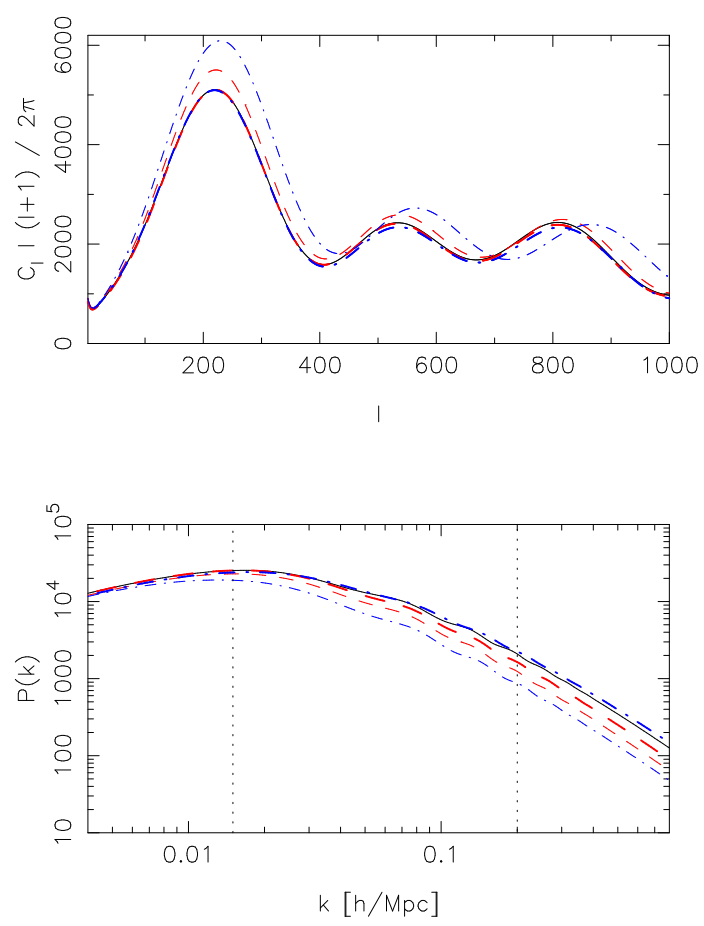

Fig. 3. Effect of extra sterile neutrino on the CMB (top) and LSS (bottom) power spectra. Thin lines correspond to standard model, sterile neutrino of mass $m=1 \mathrm{eV}$ (dashed) $m=0.3 \mathrm{eV}$ (dot-dashed) and fixed sterile density $\omega_{s}=0.01$. These curves are normalised to large scale $C_{\ell}$. Thick dashed and dot-dashed curves correspond to models, which in addition to having sterile mass have had dark matter density increased to match standard $a_{\text {eq }}$ and $h$ increased to match CMB peak positions and were normalised at the first peak. Dotted vertical lines on the bottom plot enclose the area where LSS experiments are currently sensitive to with thick line normalisations chosen to illustrate the fact that the $1 \mathrm{eV}$ model is a poorer fit than $0.3 \mathrm{eV}$ model. See text for discussion.

itously, the enhanced cold matter density also mitigates the freestreaming suppression (which scales as $\left.\omega_{c}^{-1}\right)$. At larger neutrino mass $(\sim 1 \mathrm{eV})$, additional cold matter would make $a_{\mathrm{EQ}}$ too small, so $\omega_{m}$ must be closer to 0.13 and the free-streaming suppression becomes relevant again, preventing agreement with large scale structure. This is illustrated in Fig. 3. Here we show the angular CMB anisotropy and matter power spectrum for different masses at fixed $\omega_{s}$. The suppression due to free-streaming is evident in the power spectrum and clearly becomes more severe for smaller masses. However, increasing dark matter density to match the epoch of matter-radiation equality opposes this effect. Crucial to this interpretation is the realization that the matter-radiation equality is very thoroughly measured by the present-day experiments with little model-dependence. The constraint can be summarised in $a_{\text {eq }} \sim(2.95 \pm 0.13) \times 10^{-4}$. 


\section{Conclusions}

By combining data from cosmic microwave background experiments, galaxy clustering and Ly-alpha forest observations we place new constraints on neutrino masses. In the framework of the 3 neutrino scenario cosmological data place an upper limit of $\Sigma m_{\nu}<0.17 \mathrm{eV}$ at $95 \%$ c.l.. The tension (at more than $3 \sigma$ ) between the limits from cosmology and the lower limit on $m_{\beta \beta}>0.17 \mathrm{eV}$ claimed by the Heidelberg-Moscow experiment is a clear symptom of possible problems, either in some data sets or in their theoretical interpretation, which definitely prevent any global combination of data. It would be premature to conclude that, e.g., the $0 \nu 2 \beta$ claim is "ruled out" by cosmological data but it is anyway exciting that global neutrino data analyses have already reached a point where fundamental questions may start to arise. For the 3 massless + 1 massive thermal neutrino case we bound the mass of the sterile neutrino to $m_{\nu}<0.26 \mathrm{eV}$ at $95 \%$ c.l.. Marginalizing over active neutrino masses improves the limit to $m_{\nu}<0.23 \mathrm{eV}$. These limits are incompatible at more than $3 \sigma$ with the LSND result $0.6 \mathrm{eV}^{2}<\Delta \mathrm{m}_{\mathrm{LSND}}^{2}<2 \mathrm{eV}^{2}$ (95\% C.L.). Moreover, our analysis renders the LSND anomaly incompatible at high significance with a degenerate active neutrino scenario and viceversa. If we allow for the possibility of a non-thermal sterile neutrino, we find that the upper limit of allowed energy density in the sterile neutrino is a strong function of mass. In particular, for $m_{s}<1 \mathrm{eV}$ or $>0.05 \mathrm{eV}$ the cosmological energy density in sterile neutrinos is always constrained to be $\omega_{s}<0.003$, but that for sterile neutrino mass of $\sim 0.25 \mathrm{eV}, \omega_{s}$ can be as large as $0.01 \mathrm{eV}$.

The authors would like to thank the organizers of the Workshop, Asantha Cooray and Manoj Kaplinghat. Many thanks also to G. Fogli, E. Lisi, A. Marrone, P. McDonald, A. Palazzo, U. Seljak and J. Silk.

\section{References}

[1] V. Barger, D. Marfatia and K. Whisnant, Int. J. Mod. Phys. E 12, 569 (2003).

[2] A.D. Dolgov, Phys. Rept. 370, 333 (2002).

[3] WMAP Collaboration, C.L. Bennett et al., Astrophys. J. Suppl. 148, 1 (2003).

[4] SDSS Collaboration, M. Tegmark et al., Phys. Rev. D 69, 103501 (2004).

[5] Review of Particle Physics, S. Eidelman et al., Phys. Lett. B 592, 1 (2004).

[6] W. Hu, D.J. Eisenstein, and M. Tegmark, Phys. Rev. Lett. 80, 5255 (1998).

[7] C.P. Ma and E. Bertschinger, Astrophys. J. 455, 7 (1995).

[8] SDSS Collaboration, P. McDonald et al., astro-ph/0405013. 
[9] U. Seljak et al., astro-ph/0407372.

[10] G. L. Fogli, E. Lisi, A. Marrone, A. Melchiorri, A. Palazzo, P. Serra and J. Silk, Phys. Rev. D 70 (2004) 113003 [arXiv:hep-ph/0408045].

[11] G. L. Fogli, E. Lisi, D. Montanino, and A. Palazzo, Phys. Rev. D 65, 073008 (2002).

[12] B.H.J. McKellar, Phys. Lett. B 97, 93 (1980); F. Vissani, Nucl. Phys. B (Proc. Suppl.) 100, 273 (2001); J. Studnik and M. Zralek, hepph/0110232. See also the discussion in Y. Farzan and A.Yu. Smirnov, Phys. Lett. B 557, 224 (2003).

[13] C. Weinheimer, Nucl. Phys. Proc. Suppl. 118, (2003) 279.

[14] V.M. Lobashev, in the Proceedings of NPDC 17, ed. by N. Auerbach, Zs. Fulop, Gy. Gyurky, and E. Somorjai, Nucl. Phys. A 719, 153 (2003).

[15] S.M. Bilenky, J. Hosek, and S.T. Petcov, Phys. Lett. B 94, 495 (1980); J. Schechter and J.W.F. Valle, Phys. Rev. D 22, 2227 (1980).

[16] H.V. Klapdor-Kleingrothaus, A. Dietz, H.L. Harney, and I.V. Krivosheina, Mod. Phys. Lett. A 16, 2409 (2001).

[17] H.V. Klapdor-Kleingrothaus, A. Dietz, I.V. Krivosheina, and O. Chkvorets, Nucl. Instrum. Meth. A 522, 371 (2004).

[18] H.V. Klapdor-Kleingrothaus, I.V. Krivosheina, A. Dietz, and O. Chkvorets, Phys. Lett. B 586, 198 (2004).

[19] A. Aguilar et al., Phys. Rev., D64, 112007, 2001, hep-ex/0104049.

[20] S. Dodelson, A. Melchiorri and A. Slosar, arXiv:astro-ph/0511500.

[21] G. Fogli, E. Lisi, A. Marrone, A. Melchiorri, A. Palazzo, A. Slosar, J. Silk, in preparation

[22] A. Lewis and S. Bridle, Phys. Rev. D 66, 103511 (2002) (Available from http://cosmologist.info.)

[23] D. N. Spergel et al., arXiv:astro-ph/0603449.

[24] G. Hinshaw et al., arXiv:astro-ph/0603451.

[25] W.L. Freedman et al., Astrophys. J. 553, 47 (2001).

[26] Riess et al., Astrophys. J. 665R, 607, (2004).

[27] MacTavish et al., arXiv:astro-ph/0507503.

[28] Readhead et al., ApJ, 609, 498, 2004.

[29] C. Dickinson et al., MNRAS,353, 732, 2004.

[30] C.-L. Kuo et al., American Astronomical Society Meeting, 201, 2002.

[31] M. Tegmark et al., ApJ, 606,702,2004.

[32] S. Cole et al., MNRAS, 362, 505, 2005.

[33] P. Astier et al., A\&A, 447,31, 2006.

[34] D. J. Eisenstein et al., ApJ, 633, 560, 2005.

[35] P. McDonald et al., Astrophys. J. Suppl., 163, 80, 2006, astro$\mathrm{ph} / 0405013$.

[36] P. McDonald et al., Astrophys. J., 635, 761, 2005, astro-ph/0407377.

[37] U. Seljak et al., 2006, astro-ph/0604335.

[38] M. Fukugita, K. Ichikawa, M. Kawasaki and O. Lahav, arXiv:astro$\mathrm{ph} / 0605362$. 